\title{
ON THE ADDITION THEOREM FOR MULTIPLY PERIODIC FUNCTIONS
}

\author{
S. BOCHNER
}

We shall comment on the following classical proposition.

THEOREM 1. (i) The Weierstrass function satisfies a relation

$$
P_{0}(\wp(z+w), \wp(z), \wp(w))=0,
$$

identically in independent variables, $z$, w, where $P_{0}(\alpha, \beta, \gamma)$ is a polynomial not $\equiv 0$ with constant coefficients.

(ii) More generally, any doubly periodic meromorphic function $f(z)$ satisfies a polynomial relation

$$
P_{00}(f(z+w), f(z), f(w))=0 .
$$

(iii) Even more generally, for any three such functions

$$
f(z), \quad \phi(z), \quad \psi(z)
$$

with the same periods, the last two not $\equiv c$, we have a relation

$$
P(f(z+w), \phi(z), \psi(w))=0 .
$$

(iv) Also, $\varphi(z+w)$ is a rational function of $\varphi(z), \varphi^{\prime}(z), \wp(w), \varphi^{\prime}(w)$; and more generally if $\phi^{1}(z), \phi^{2}(z)$ are rationally independent [as $\wp(z), \varphi^{\prime}(z)$ are $]$ and $\psi^{1}(w), \psi^{2}(w)$ are rationally independent, then $f(z+w)$ is a rational function of $\phi^{1}(z), \phi^{2}(z), \psi^{1}(w), \psi^{2}(w)$.

On the face of it, part (i) is a particular case of part (ii), and the latter part of (iii), but actually all three parts are algebraically equivalent by the following elementary argument. For given periods, we introduce the closed Riemann surface $V_{2}$ of genus $p=1$ on which our meromorphic functions are suitably defined, and we take it as known that on such or any other closed Riemann surface of any genus $p \geqq 0$, any two meromorphic functions are algebraically dependent one on the other. That is to say, if for any $f(z) \neq c$ on $V_{2}$ we introduce the function field

$$
K[f(z)]
$$

over complex constants, then any other $g(z)$ is algebraic over (5), thus satisfying a polynomial relation $P^{0}(f(z), g(z))=0$. In particular, the functions (3) are connected with the Weierstrass function by relations

Received by the editors January 25, 1951. 


$$
\begin{gathered}
P_{1}(\varphi(z+w), f(z+w))=0, \quad P_{2}(\varphi(z), \phi(z))=0, \\
P_{3}(\varphi(w), \psi(w))=0,
\end{gathered}
$$

and if we start out from (1) then formal algebraic elimination as between (1) and (6) will produce (4). A similar argument will equate the two halves of part (iv). Also a suitable way of expressing (4) is to say that the two-variable function

$$
f(z, w)=f(z+w),
$$

whose domain of existence is the product $V_{2} \times V_{2}$ of our torus with itself, is algebraic over the function field

$$
K[\phi(z) ; \psi(w)],
$$

and the second half of (iv) states that it is contained in

$$
K\left[\phi^{1}(z), \phi^{2}(z) ; \psi^{1}(w), \psi^{2}(w)\right] .
$$

An "addition theorem" suggests colorful connections to arithmetic, and such are indeed manifest in statements of many facets. The point we wish to make, however, is this-that to our theorem, as stated, no such connection is as yet relevant and that it is a special case of a vastly more general and entirely colorless proposition in which additivity is in no way referred to.

If we take any doubly periodic periodic meromorphic function $f(z)$, and introduce the two-variable function (7), then for each fixed $w$ (with some possible exceptions) the latter is again such a function in $z$, and for each fixed $z$ it is such a function in $w$, and this is all that is needed to validate our conclusions. In fact, if we realize that for any three (or more) meromorphic functions on $V_{2}$ there is a point of the space in the neighborhood of which they are all holomorphic (without any polar singularities), then Theorem 1 is obviously contained in the following "local" theorems which we shall immmediately state for multi-dimensional variables as well.

Theorem 2. If $A$ is a domain in the space of $k$ complex variables $z=\left(z^{(1)}, \cdots, z^{(k)}\right)$ and $B$ is a domain in the space of $l$ complex variables $w=\left(w^{(1)}, \cdots, w^{(l)}\right)$; if we are given a finite number of holomorphic functions

$$
\phi^{1}(z), \cdots, \phi^{r}(z)
$$

in $A$, and a finite number of holomorphic functions

$$
\psi^{1}(w), \cdots, \psi^{*}(w)
$$

in $B$; and if a holomorphic function $f(z, w)$ in $A \times B$ has the property 
that for each w in $B$ it is algebraic over

$$
K\left[\phi^{1}(z), \cdots, \phi^{r}(z)\right]
$$

and for each $z$ in $A$ it is algebraic over

$$
K\left[\psi^{1}(w), \cdots, \psi^{*}(w)\right]
$$

then in $A \times B$ it is algebraic over

$$
K\left[\phi^{1}(z), \cdots, \phi^{r}(z) ; \psi^{1}(w), \cdots, \psi^{s}(w)\right] .
$$

TheOREM 3. If the function $f(z, w)$ is for each $w$ in $B$ contained in the field (12) and for each $z$ in $A$ in the field (13), then in $A \times B$ it is contained in the field (14).

There is also a mixed theorem as follows.

TheORem 4. If $f(z, w)$ is for each w algebraic over (12), of a degree not greater than $g$, where $g$ is independent of $w$, and if for each $z$ it is contained in (13), then in $A \times B$ it is algebraic over (14), of degree not greater than $g$.

A particular consequence of Theorems 2 and 3 is the following known generalization of Theorem 1 to many dimensions. ${ }^{1}$

THEOREM 5. If for $k$ complex variables there are given $k$ algebraically independent meromorphic functions

$$
\phi^{1}(z), \cdots, \phi^{k}(z)
$$

with the same $2 k$ "independent" periods, then for any other such function $f(z)$-which in particular may be one of the $\phi p(z)$-the new function $f(z+w)$ is algebraic over

$$
\dot{K}\left[\phi^{1}(z), \cdots, \phi^{k}(z) ; \phi^{1}(w), \cdots, \phi^{k}(w)\right] ;
$$

and if there are given $k+1$ rationally independent functions

$$
\phi^{0}(z), \phi^{1}(z), \cdots, \phi^{k}(z),
$$

then $f(z+w)$ is rational over

$$
K\left[\phi^{0}(z), \cdots, \phi^{k}(z) ; \phi^{0}(w), \cdots, \phi^{k}(w)\right] .
$$

Although syllogistically subordinate to Theorems 2, 3, our Theorem 5 has nevertheless the distinguishing feature that in it the twovariable function occurring is actually a one-variable function in which the variable $z$ has been replaced by the two-fold argument

\footnotetext{
${ }^{1}$ A. Krazer and W. Wirtinger, Abelsche Funktionen und allgemeine Thetafunktionen, Encyclopädie der mathematischen Wissenschaften II B 7, p. 827.
} 
$z+w$. Now this distinguishing feature has a generalization of its own and it is as follows.

Let the domain $A$ of our theorems be a coordinate neighborhood of a complex manifold $V_{2 k}$; let $W_{2 l}$ be a (complex) Lie group acting on $V_{2 k}$, and let $B$ be a neighborhood of the identity in $W_{2 l}$. Now, if $f(z)$ is meromorphic on $V_{2 k}$ and has no singularities in $A$, and if $w$ is a point of $B$, and if we denote by $f(w(z))$ the function resulting from carrying out the homeomorphism " $w$," then we obviously obtain a two-variable function $f(z, w)$ on $A \times B$ to which our Theorems 2, 3, 4 can be applied, provided, of course, that finite systems of functions $\left\{\phi^{\rho}(z)\right.$; $\left.\psi^{\sigma}(w)\right\}$ as prescribed in the theorems are available on $A, B$.

A pertinent though seemingly "trivial" illustration arises if for $V_{2 k}$ we take the ordinary Gaussian sphere $V_{2}$ and for $W_{2 l}$ the group $W_{6}$ of projective transformations

$$
z^{\prime}=\frac{w^{\prime}+w^{\prime \prime} z}{w^{\prime \prime}+w^{\prime \prime \prime} z}
$$

on it. A meromorphic function on $V_{2}$ is any ordinary rational function in $z$,

$$
f(z)=R(z) \equiv \frac{\sum a_{\mu} z^{\mu}}{\sum b_{\nu} z^{\nu}}
$$

and the quantities $w^{\prime}, w^{\prime \prime}, w^{\prime \prime \prime}, w^{\prime \prime \prime \prime}$ are obviously meromorphic functions on the rational surface

$$
w^{\prime} w^{\prime \prime \prime \prime}-w^{\prime \prime} w^{\prime \prime \prime}=1
$$

constituting $W_{6}$. Now, the two-variable function

$$
f(z, w)=R\left(\frac{w^{\prime}+w^{\prime \prime} z}{w^{\prime \prime \prime}+w^{\prime \prime \prime \prime} z}\right)
$$

has the property that for each $w$ it is meromorphic in $z$ and for each $z$ meromorphic in $w$ and, in conformity with Theorem 3 , it is indeed meromorphic in $(z, w)$. Also, if $f(z)$ is algebraic of a degree $g$, then for each $w, f(z, w)$ is algebraic of a degree not greater than $g$, and for each $z$ it is rational in $w$, and, in conformity with Theorem 4 , it is indeed algebraic in $(z, w)$ of degree $g$.

The example obviously falls under the following assertions. $(\alpha)$ If the holomorphic function $f(z, w)$ in $A \times B$ is for each $w$ algebraic in the local parameters $z$, and for each $z$ algebraic in the parameters $w$, then it is algebraic in $(z, w)$. ( $\beta$ ) If it is rational in $z$ and $w$ separately it is rational in them jointly. $(\gamma)$ If for each $w$ it is algebraic in $z$ of 
degree not greater than $g$, and for each $z$ rational in $w$, then it is algebraic in $(z, w)$ of degree not greater than $g$. Now, assertion $(\beta)$ was first stated by Weierstrass, ${ }^{2}$ and then proven by A. Hurwitz $;^{3}$ and assertions $(\alpha)$ and $(\gamma)$ were set up and proven by ourselves ${ }^{4}$ when reproducing the proof of Hurwitz in the simplified version given to it by $\mathrm{H}$. Kneser ; ${ }^{5}$ and the proofs to follow of the general Theorems 2, 3, 4 will be nearly literally the same as those given in the paper cited in footnote 4 for the particular assertions $(\alpha),(\beta),(\gamma)$. But before giving the proofs we wish to point out that although the problem of Weierstrass and Hurwitz dealt directly only with meromorphic functions of the ordinary kind, that is to say, with functions on complex projective spaces, yet the context in which it arose was one dealing with multi-periodic functions and Abelian integrals primarily; so that our general versions of the theorems may be said to be related to the original ones not only syllogistically but even genetically as well, although our proof itself would hardly betoken such a development.

The proof of our theorems rests on the following lemma, which by itself does not yet refer to analyticity or even continuity.

Lemma 1. If $F_{1}(z, w), \cdots, F_{N}(z, w)$ are functions in $A \times B$, not all $\equiv 0$, and if they satisfy a relation

$$
c_{1}(w) F_{1}(z, w)+\cdots+c_{N}(w) F_{N}(z, w)=0
$$

with arbitrary coefficients $\left\{c_{n}(w)\right\}$ for which

$$
\left|c_{1}(w)\right|^{2}+\cdots+\left|c_{N}(w)\right|^{2}>0,
$$

then they also satisfy such a relation with other coefficients $C_{n}(w)$, not all $\equiv 0$, from the smallest function ring over complex coefficients containing all functions $\left\{F_{n}(a, w)\right\}$ for all special values $z=a$ and $n=1, \cdots, N$.

In particular if each $F_{n}(z, w)$ belongs to (13), then there are coefficients $C_{n}(w)$ belonging likewise to (13).

${ }^{2} \mathrm{C}$. Weierstrass, Untersuchungen über die $2 r$-fach periodischen Functionen von $r$ Veränderlichen, J. Reine Angew. Math. vol. 89 (1880) pp. 1-8; or, Werke, vol. 2, pp. 125-133.

${ }^{3}$ A. Hurwitz, Beweis des Satzes, dass eine einwertige Function beliebig vieler Variabeln, welche überall als Quotient zweier Potenzreihen dargestellt werden kann, eine rationale Function ihrer Argumente ist, J. Reine Angew. Math. vol. 95 (1883) pp. 201206.

S. Bochner and W. T. Martin, Several complex variables, Princeton, 1948, pp. 199-203.

5 H. Kneser, Einfacher Beweis eines Satzes über rationale Funktionen Zweier Veränderlschen, Abh. Math. Sem. Hamburgischen Univ. vol. 9 (1933) pp. 195-196. 
Proof. We write relation (22) for $N$ values $z_{1}, \cdots, z_{N}$, thus obtaining a system of $N$ homogeneous equations in the quantities $c_{n}(w)$. Because of (23), the determinant

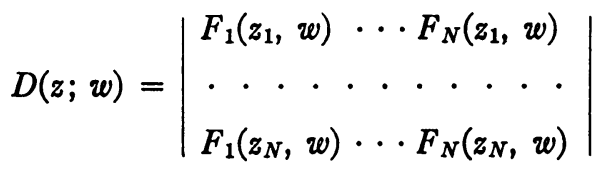

must vanish identically in $w \in B ; z_{1} \in A, \cdots, z_{N} \in A$. We now replace the letter $z_{N}$ by $z$, land develop the determinant in terms of its last row. This leads to a relation

$$
\sum_{n=1}^{N} C_{n}\left(z_{1}, \cdots, z_{N-1} ; w\right) F_{n}(z, w)=0
$$

in which the functions

$$
C_{n}\left(z_{1}, \cdots, z_{N-1} ; w\right)
$$

are, but for \pm signs, the $(n-1)$-dimensional determinants of the matrix

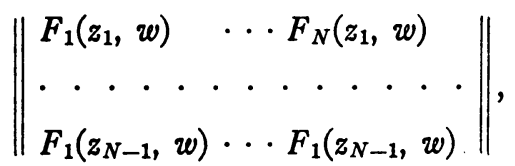

and we first assume that not all functions (26) vanish identically in all variables. Hence there exist numerical values $z_{1}=a_{1}, \cdots, z_{N-1}$ $=a_{N-1}$ such that the functions

$$
C_{n}(w)=C_{n}\left(a_{1}, \cdots, a_{N-1} ; w\right)
$$

do not vanish identically in $w$, and for such numerical values relation (25) reduces to relation (22) with new coefficients $c_{n}(w)$ having the property stated in the lemma. If, however, all functions (26) vanish identically, then the determinant (24) has a principal minor

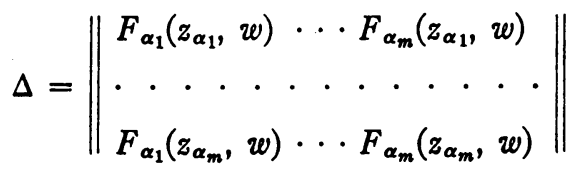

which vanishes identically, but which has at least one subdeterminant of order $m-1$ which does not vanish identically. By the result just proved there exists a relation

$$
\sum_{\mu=1}^{m} C_{\alpha \mu}(w) F_{\alpha \mu}(z, w)=0,
$$


and by inserting vanishing coefficients $C_{n}(w)$ we obtain the conclusion.

Proof of Theorem 3. We form the products

$$
\left(\phi^{1}(z)\right)^{n_{1}} \cdots\left(\phi^{r}(z)\right)^{n_{r}}
$$

for all combinations of integers $n_{1} \geqq 0, \cdots, n_{r} \geqq 0$ and denote the resulting sequence of "monomials" in some ordering by $p_{0}(z)$, $p_{1}(z), \cdots$. By assumption of the theorem we have for each $w$ a relation

$$
\left(\sum_{\mu=0}^{m} a_{\mu}(w) p_{\mu}(z)\right) f(z, w)+\sum_{\nu=0}^{n} b_{\nu}(w) p_{\nu}(w)=0
$$

with $m, n$ depending on $w$, and

$$
\sum_{\mu=0}^{m}\left|a_{\mu}(w)\right|^{2}+\sum_{\nu=0}^{n}\left|b_{\nu}(w)\right|^{2}>0,
$$

and if we multiply it by a factor we may presuppose the normalization

$$
\sum_{\mu=0}^{m}\left|a_{\mu}(w)\right|^{2}+\sum_{\nu=0}^{n}\left|b_{\nu}(w)\right|^{2}=1 .
$$

By the same argument as on p. 201 of the paper cited in footnote 4, there exist some combination of degrees $(m, n)$ for which relation (28) holds for a subdomain of $B$, and if we now denote the subdomain itself by $B$, then (28) will hold everywhere in $A \times B$ for a given set of degrees $(m, n)$. If now we put $N=m+n, F_{\mu}(z ; w)$ $=p_{\mu}(z) f(z, w)$ for $\mu=1, \cdots, m$, and $F_{m+\nu}(z ; w)=p_{\nu}(z)$ for $\nu=1, \cdots$, $n$, then our lemma becomes applicable, and the conclusion is that there is also a relation (28) with coefficients $a_{\mu}(w), b_{\nu}(w)$ belonging to the function ring (13), and not all $\equiv 0$, which is precisely the assertion of the theorem.

Proof of Theorems 2 AND 4. In this case we have for each $w$ a relation

$$
\sum_{\mu=0}^{m} \sum_{\gamma=0}^{o} a_{\mu \gamma}(w) p_{\mu}(z) f(z, w)^{\gamma}=0,
$$

and for some subdomain $B_{0}$ of $B$ this relation is available for the same $(m, g)$, where in the case of Theorem 4 the integer $g$ is the one specified in the theorem. We now put $N=m(g+1)$, and apply the lemma to the functions 


$$
F_{n}(z, w)=p_{\mu}(z) f(z, w)^{\gamma} .
$$

As a consequence of the lemma we obtain a new relation with the same $(m, g)$ in which the $a_{\mu \gamma}(w)$ belong to the smallest function ring containing all $p_{\mu}(a) f(a, w)^{\gamma}$, that is, all $f(a, w)^{\gamma}$, and in the case of Theorem 4 this terminates the proof immediately. In the case of Theorem 2 we have to add the step that the new coefficients are algebraic over the smallest function ring containing all $f(a, w)$, and by an elementary theorem on field extensions a second choice of coefficients $a_{\mu \gamma}(w)$, with other $(m, g)$, will produce a final relation (28) in which the coefficients $a_{\mu \gamma}(w)$ are contained in the function ring (13) itself, as claimed.

REMARK. The two function fields (12) and (13), of which (14) is the "product," have been assumed to have each a finite basis over the field of complex numbers, but the proof of the theorems just completed shows that this requirement of finiteness can be relaxed in the following way. The function field (13) of holomorphic functions on $B$ can have an arbitrary potency, and we shall denote it by $K_{w}$. The field on $A$ however-we shall denote it by $K_{z}$-cannot be allowed to be arbitrary, but it need not be finite either. Rather, it suffices to assume that it is the limit of an increasing sequence of subfields $K_{2}^{q}, q=1,2, \cdots$, of which each has a finite basis, such that, in the case of Theorem 2, our function $F(z, w)$ is for each $w$ algebraic over some $K_{z}^{q}, q=q(w)$, and contained in it in case of Theorem 4. The conclusion then is that $F(z, w)$ is algebraic, or rational, over the product $K_{w} \times K_{z}$, and indeed over $K_{w} \times K_{z}^{q_{0}}$, for some suitable index $q_{0}$ depending on the function.

Princeton University 